\title{
Site-Specific Kondo Effect at Ambient Temperatures in Iron-Based Molecules
}

\author{
L. Gao, ${ }^{1}$ W. Ji, ${ }^{1}$ Y. B. Hu, ${ }^{1}$ Z. H. Cheng, ${ }^{1}$ Z. T. Deng, ${ }^{1}$ Q. Liu, ${ }^{1}$ N. Jiang, ${ }^{1}$ X. Lin, ${ }^{1}$ W. Guo, ${ }^{1}$ S. X. Du, ${ }^{1}$ W. A. Hofer, ${ }^{2}$ \\ X. C. Xie, ${ }^{3,1}$ and H.-J. Gao ${ }^{1, *}$ \\ ${ }^{1}$ Beijing National Laboratory for Condensed Matter Physics, Institute of Physics, Chinese Academy of Sciences, P.O. Box 603, \\ Beijing 100080, China \\ ${ }^{2}$ Surface Science Research Center, The University of Liverpool, Liverpool L69 3BX, United Kingdom \\ ${ }^{3}$ Department of Physics, Oklahoma State University, Stillwater, Oklahoma 74078, USA \\ (Received 29 August 2006; revised manuscript received 10 May 2007; published 5 September 2007)
}

\begin{abstract}
Kondo resonances are a very precise measure of spin-polarized transport through magnetic impurities. However, the Kondo temperature, indicating the thermal range of stability of the magnetic properties, is very low. By contrast, we find for iron phthalocyanine a Kondo temperature in spectroscopic measurements which is well above room temperature. It is also shown that the signal of the resonance depends strongly on the adsorption site of the molecule on a gold surface. Experimental data are verified by extensive numerical simulations, which establish that the coupling between iron states and states of the substrate depends strongly on the adsorption configuration.
\end{abstract}

DOI: 10.1103/PhysRevLett.99.106402

PACS numbers: 71.15.Mb, 71.10.- $-\mathrm{w}$

Because of their importance in the emerging field of molecular electronics the transport properties of single molecules have been the focus of intensive research, leading to the concept of a single-electron transistor [1] and a proof of principle of field regulation by external charges [2]. The magnetic properties of transition metal atoms in a host molecule can be detected by a Kondo resonance in cryogenic scanning tunneling microscopes [3-5]. These properties are important variables in the fabrication of single molecule devices [6-10]. Most previous studies of the Kondo effect focused on magnetic atoms on open metal surfaces [11-21]. However, the Kondo temperature in these systems is very low; the spin-dependent transport properties are consequently lost in an ambient environment. Recent studies of molecular Kondo effects show that caging the magnetic atoms in a molecule can elevate $[3,4,22]$ or decrease $[5,22]$ the Kondo temperature. In this case, both the molecular structure [4,5] and the molecular conformation [3] play an important role. Here, we present measurements of the Kondo effect of iron phthalocyanine $(\mathrm{FePc})$ molecules on an $\mathrm{Au}(111)$ surface. The results are significant for two reasons. First, they indicate a high Kondo temperature, well above room temperature, for the FePc molecule adsorbed on $\mathrm{Au}(111)$ surface. This is in marked contrast to the low Kondo temperature for isolated $\mathrm{Fe}$ atoms adsorbed on the same surface [17]. Second, they reveal a substantial change of the effect with the adsorption configuration of the molecule. This indicates that magnetic properties of an interface can be controlled at the molecular level by site-specific adsorption.

The scanning tunneling microscopy (STM) experiments were performed on an $\mathrm{Au}(111)$ single crystal surface, prepared carefully by repeated cycles of $\mathrm{Ar}^{+}$sputtering and annealing in ultrahigh vacuum (base pressure $3 \times$ $10^{-10}$ mbar). FePc molecules were evaporated from the sublimation cell onto the gold surface at a temperature of $\sim 370 \mathrm{~K}$. Then the sample was transferred in situ into an
Omicron low-temperature STM operating at $4.6 \mathrm{~K}$. The bias voltage is applied to the sample. $d I / d V$ spectra were measured using a lock-in technique with a modulation signal $\left(4 \mathrm{mV}_{\mathrm{rms}}, 1 \mathrm{kHz}\right)$. The tungsten tip has been carefully prepared before spectroscopic measurements to ensure no tip-related features on the recorded $d I / d V$ spectra. A topographic image of the $\mathrm{Au}(111)$ surface, covered by $\sim 2 \times 10^{13}$ per $\mathrm{cm}^{2} \mathrm{FePc}$ molecules, is shown in Fig. 1 . The image of a single FePc molecule is a "cross" with a bright spot at the center, indicating a flat-lying adsorption configuration. The enhanced brightness at the molecular

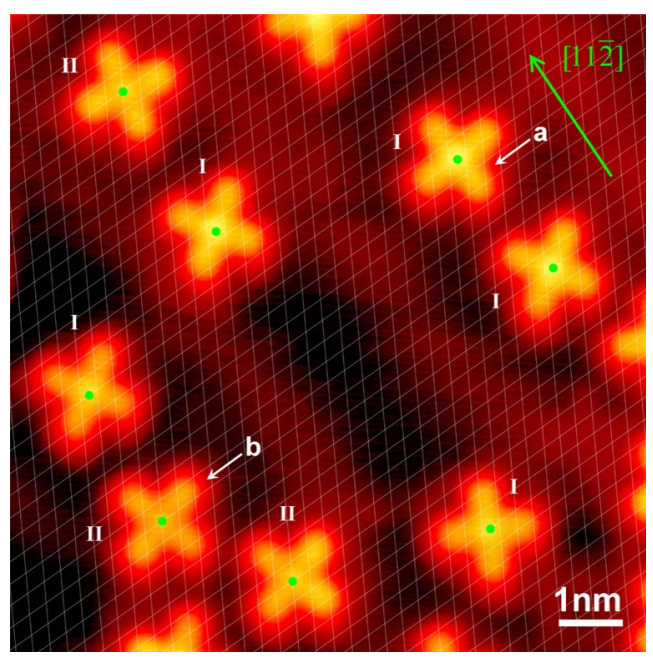

FIG. 1 (color online). STM image of isolated FePc molecules on $\mathrm{Au}(111)$ surface. Scanning parameters: $10 \mathrm{~nm} \times 10 \mathrm{~nm}, U=$ $-0.5 \mathrm{~V}, I=69 \mathrm{pA}$. The direction of the $\mathrm{Au}(111)$ substrate is determined by surface reconstruction. The overlayed grid represents the gold substrate lattice, showing a shift of $1 / 2$ unit cell between the adsorption sites of the two types (I and II) of molecules. The $d I / d V$ curves in (a) and (b) of Fig. 2 are measured at the center of molecules marked with (a) and (b), respectively. 
center is ascribed to the $d$-orbital character of the $\mathrm{Fe}(\mathrm{II}) d^{6}$ system near the Fermi level $[23,24]$. We found two molecular adsorption configurations on the $\mathrm{Au}(111)$ substrate. For one configuration, called configuration I, the cross is

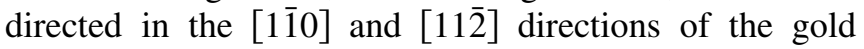
substrate; for the other configuration, called configuration II, the cross rotates with respect to the molecular center by $\sim 15^{\circ}$ compared to configuration I.

Differential conductance $d I / d V$ spectra were measured precisely at the molecular center. A scan near the Fermi level $\left(V_{\text {sample }}=0\right)$ shows that the two adsorption configurations have different features in the $d I / d V$ spectra. The $d I / d V$ spectra show a narrow peak for configuration I [see Fig. 2(a)], but a narrow dip for configuration II [see Fig. 2(b)]. The relation of the difference between the peak and the dip to the adsorption configuration has been verified by measuring over 100 molecules. The peak and dip features are based on a background slope in the $d I / d V$ spectra. $d I / d V$ spectra with a larger energy range [Fig. 2(c)] show that the background slope is the result of broad resonances. For configuration I, besides the narrow peak FR-I, there are two other broad resonances, Fe-I and SS-I, located at $\sim 760 \mathrm{meV}$ and $\sim 230 \mathrm{meV}$ below the Fermi level, respectively. For configuration II, besides the narrow dip FR-II, the other two broad resonances, Fe-II and SS-II, are located at $\sim 860 \mathrm{meV}$ and $\sim 230 \mathrm{meV}$ below the Fermi level, respectively. The resonance widths and the electronic structure of FePc indicate that the broad resonances Fe-I and Fe-II are $d$ orbitals of Fe atom [25]. The low energy resonances (SS-I, SS-II) might originate from the surface state of the gold substrate. The line shape of the $d I / d V$ spectra near the Fermi level can be fitted to a Fano function $[21,22,26-32]$.
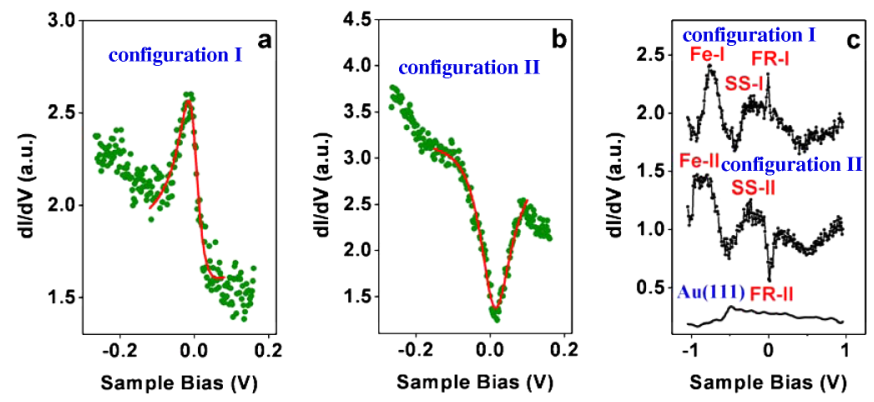

FIG. 2 (color online). (a) $d I / d V$ spectra (dotted line) for configuration I, measured at the center of FePc molecule (a in Fig. 1), showing a peak near the Fermi level. The solid line in the spectra is a fit of a Fano function. The fit parameters are $q=$ $2.20 \pm 0.19, \quad \varepsilon_{0}=1.58 \pm 0.82 \mathrm{meV}, \quad \Gamma=30.73 \pm 1.77 \mathrm{meV}$. (b) $d I / d V$ spectra (dotted line) for configuration II, measured at the center of FePc molecule [(b) in Fig. 1], showing a dip near the Fermi level. The solid line in the spectra is a fit of a Fano function. The fit parameters are $q=0.12 \pm 0.03, \varepsilon_{0}=-8.39 \pm$ $0.63 \mathrm{meV}, \Gamma=51.52 \pm 1.60 \mathrm{meV}$. (c) $d I / d V$ spectra scanned with a wider energy range, measured at the center of FePc molecules. Fe-I and Fe-II are the Fe-3 $d$ state; SS-I and SS-II are the surface state; FR-I and FR-II are the Fano resonances.

$$
\frac{d I(V)}{d V}=A \frac{(\varepsilon+q)^{2}}{1+\varepsilon^{2}}+B
$$

with $\varepsilon=\left(e V-\varepsilon_{0}\right) / \Gamma$. In this equation, $A$ is the amplitude coefficient, $B$ is the background $d I / d V$ signal, $q$ is the Fano line shape parameter, $\varepsilon_{0}$ is the energy shift of the resonance from the Fermi level, and $\Gamma$ is the half width of the resonance.

The most likely explanation for the peak and dip features near the Fermi level is that they are signatures of a Kondo resonance. This interpretation is supported by the following arguments. First, the line shape near the Fermi level excludes the possibility of a $d$-orbital resonance. Second, the width of the peak is significantly smaller than the resonance width of $d$ orbitals [see Fig. 2(c)]. Third, the energy locations for the peak and the dip features are very close to the Fermi level. In addition, only $d I / d V$ spectra measured at the molecular center show the peak or dip features near the Fermi level, which indicates that the magnetic atom is the origin of the peak and dip features in the $d I / d V$ spectra. An additional signature of a Kondo resonance is the temperature dependence of the resonance width. However, due to thermal effects it is close to impossible to obtain stable molecular STM images above $20 \mathrm{~K}$. The fitted Fano resonance width $\Gamma$ yields the Kondo temperature $T_{K}$ due to $k_{B} T_{K}=\Gamma$. We find that the Kondo temperature $T_{K}$ is $357 \pm 21 \mathrm{~K}$ for configuration I and $598 \pm 19 \mathrm{~K}$ for configuration II. To explain the difference in both line shape and the Kondo temperature between the two configurations, it is necessary to identify the precise configurations for experimentally observed two adsorption configurations.

To this end we carried out first-principles calculations based on density functional theory (DFT), a PerdewBurke-Ernzerhof (PBE) generalized gradient approximation for the exchange-correlation energy [33], projector augmented waves (PAW) [34,35], and a plane wave basis set as implemented in the Vienna ab initio simulation package (VASP) [36,37]. Because of numerical limitations and the size of the system the surface Brillouin zone was sampled with the $\bar{\Gamma}$ point only. The cutoff energy for the plane waves was $400 \mathrm{eV}$. In structural relaxations, all atoms except for the bottom three Au layers were fully relaxed until the net force on every atom was smaller than $0.02 \mathrm{eV} / \AA ̊ . \mathrm{A} c(7 \times 8)$ supercell was employed to model the isolated molecule. The relaxation was initially carried out with a three-layer Au slab, separated by a vacuum layer of $12 \mathrm{Au}$ layers. Subsequently, the optimized results were checked with a four-layer slab model. The procedure was necessary due to numerical limitations: a four-layer slab contains over 280 atoms including 57 atoms of a FePc molecule, which is at the very limit of computational capacity in present PAW-DFT calculations. Eight possible adsorption configurations were considered for energy optimization in the calculations. Our results reveal that the optimized fully relaxed structure based on the configuration in Fig. 3(b) and that in Fig. 3(a) have the highest 


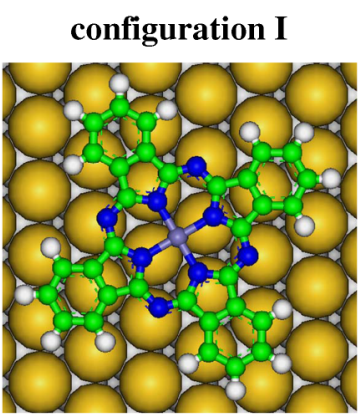

a

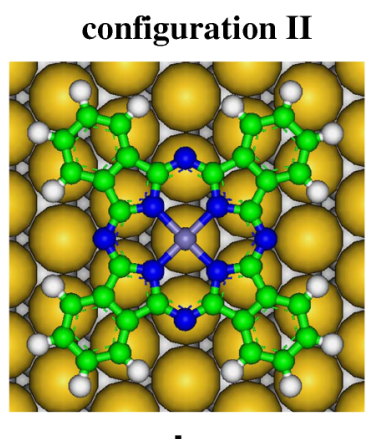

b
FIG. 3 (color online). Optimized adsorption configurations for experimentally observed configuration I (a) and configuration II (b), respectively.

adsorption energy values. The adsorption energy of the configuration in Fig. 3(b) is $104 \mathrm{meV}$ higher than the one in Fig. 3(a). And the latter is 33 to $136 \mathrm{meV}$ higher than the other possible configurations.

We checked the simulations for consistency by eight separate simulations using the Becke-Lee-Yang-Parr hybrid functional [38]. The calculations of eight possible configurations also show that the most stable configuration is the one in Fig. 3(b), followed by the one in Fig. 3(a). The adsorption energy of the configuration in Fig. 3(b) is $58 \mathrm{meV}$ higher than that in Fig. 3(a). And the latter is 36 to $477 \mathrm{meV}$ higher than the values for the other possible configurations.

Both calculation methods indicate that the configurations in Figs. 3(a) and 3(b) are the experimentally observed configuration I and configuration II, respectively. The Fe atom in configuration $I$ is at a bridge site, while in configuration II it is at the top site. The results are in agreement with experimental measurements. There is a shift of $1 / 2$ unit cell between the adsorption sites of the two configurations [Fig. 1], which in conjunction with simulations allows only one conclusion: the two configurations correspond to a top site and a bridge site, respectively. If the molecular symmetry is taken into account, there are two types of bridge sites for configuration I, both of which can be observed in experiments [Fig. 1]. However, the $d I / d V$ spectra at the molecular center for the two bridge sites of configuration I are almost the same, which reflects that the Kondo resonance is mainly dominated by the adsorption sites of the central Fe atoms.

In the following discussion we consequently limit the analysis to the two configurations shown in Fig. 3. Figure 4(c) is the calculated spin-polarized partial density of states (SP-PDOS) of the Fe atom, showing that spin-up $(\uparrow)$ states and spin-down $(\downarrow)$ states are not filled symmetrically for both configurations. The magnetic moment is $\sim 1.9 \mu_{B}$. A Kondo effect is induced by the coupling between a localized spin in an impurity orbital and a surrounding sea of conduction electrons. The Kondo temperature strongly depends both on the $d$-orbital distribution near the Fermi level, and on the strength of the spin-
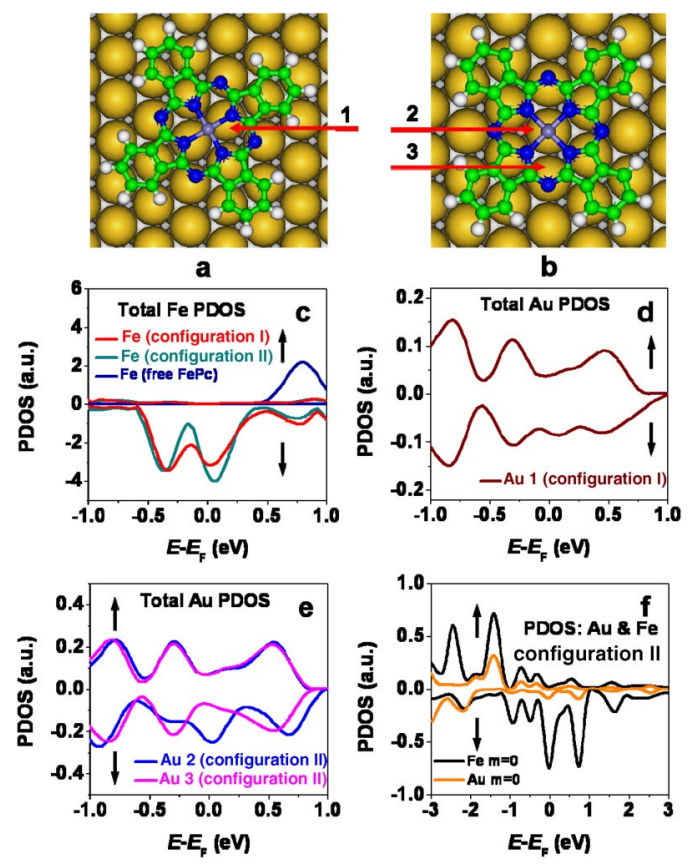

FIG. 4 (color online). SP-PDOS of $\mathrm{Fe}$ and $\mathrm{Au}$ atoms in $\mathrm{FePc} / \mathrm{Au}(111)$ systems. (a) configuration I. (b) configuration II. (c) SP-PDOS of $\mathrm{Fe}$ atoms in configuration I, configuration II, and a free FePc molecule. (d) SP-PDOS of an Au atom in configuration $\mathrm{I}$. The $\mathrm{Au}$ atom is marked with 1 in (a). (e) SP-PDOS of two Au atoms in configuration II. The Au atoms are marked with 2 and 3 in (b). (f) SP-PDOS ( $m=0$ ) of Fe atom and $\mathrm{Au}$ atom in configuration II. The $\mathrm{Au}$ atom is marked with 2 in (b).

electron coupling. The observed high Kondo temperature in the $\mathrm{FePc} / \mathrm{Au}(111)$ system is due to the significant $d$-orbital character of electrons near the Fermi level within the $\mathrm{Fe}(\mathrm{II}) d^{6}$ system [23], and to the enhanced spinelectron coupling as an effect of molecular bonding.

Our results show that there is a big difference in Kondo temperature between configuration I and configuration II. For the two configurations, the $d$-orbital distribution near the Fermi level is roughly equivalent except for a slight energy shift [see Fig. 2(c) and 4(c)], but the strength of the spin-electron coupling is quite different. The conduction electrons on the gold substrate and within the FePc molecules act as the surrounding conduction electrons in the Kondo effect. The coupling between the localized spin and substrate conduction electrons in configuration II is much stronger than that in configuration I. The calculated SPPDOS shows that the Au atom adjacent to the $\mathrm{Fe}$ atom in configuration II possesses a nonvanishing magnetic moment [Fig. 4(e)], while the adjacent Au atoms in configuration I carries no magnetic moment [Fig. 4(d)]. Thus the $\mathrm{Fe}-\mathrm{Au}$ interaction is much stronger in configuration II than in configuration I. Figure 4(f) shows obvious $d$-level hybridization between $\mathrm{Fe}$ and $\mathrm{Au}$ atoms in configuration II, which confirms this view. So the much stronger spinelectron coupling results in a much higher Kondo temperature for configuration II. 
The difference in the line shape of Kondo resonance between configuration I and configuration II is caused by the different competition between two electron tunneling channels. Within the Fano theory the line shape of the $d I / d V$ spectra for a Kondo resonance is determined by the line shape parameter $q$ [26]. $q$ describes the ratio of the probability for tunneling through magnetic impurity orbitals, and tunneling directly into the surrounding conduction band. Our experimental results shows that configuration I $(q=2.20)$ has a much larger $q$ value than configuration II $(q=0.12)$. In the $\mathrm{FePc} / \mathrm{Au}(111)$ system, the first tunneling channel is via the $d$ orbitals of the central $\mathrm{Fe}$ atom; the second channel is directly tunneling into the conduction band of the surrounding conduction electrons both on the gold substrate and within the FePc molecules. First, the molecular bonding to the substrate is stronger for configuration II than for configuration I, so it is much easier for the STM tip to detect the conduction electrons in configuration II than in configuration I. More electrons tunnel directly into the conduction band in configuration II than in configuration I. Secondly, the tunneling through central $\mathrm{Fe}$ atom is much decreased in configuration II compared to configuration I, which is obvious from low-bias STM images. In Fig. 1, the brightness of the central $\mathrm{Fe}$ atom for configuration II is much lower than that for configuration I. Therefore, different adsorption configurations have induced different interaction between $d$ orbitals and the substrate, which influences the electron tunneling through the central $\mathrm{Fe}$ atom. Compared to configuration I the electron transfer directly into the conduction band is increased, and the transfer through the central $\mathrm{Fe}$ atom is decreased in configuration II, accounting for the large difference in the line shape of the Kondo resonance.

In summary, we have observed an unusually high Kondo temperature in the $\mathrm{FePc} / \mathrm{Au}(111)$ system at the single molecular scale using LT-STM/STS. Our results show that both the Kondo temperature and the line shape of $d I / d V$ spectra are greatly influenced by molecular adsorption configuration on the Au(111) substrate. This implies that it is feasible to control the local spin coupling and the competition between different tunneling channels in molecular Kondo effect by changing the molecular adsorption configuration. Given that the lateral structure of a molecular interface can be modified by the attachment of ligands [39], the finding opens up the possibility to tailor magnetic properties of an organic interface to the desired specifications.

This project is supported partially by the NSFC and MOST 973, China. W. A. H. thanks the Royal Society for financial support. X.C.X. is supported by U.S. DOE and NSF.

*hjgao@aphy.iphy.ac.cn

[1] S. Kubatkin et al., Nature (London) 425, 698 (2003).

[2] P. G. Piva et al., Nature (London) 435, 658 (2005).
[3] V. Iancu, A. Deshpande, and S. W. Hla, Nano Lett. 6, 820 (2006).

[4] P. Wahl et al., Phys. Rev. Lett. 95, 166601 (2005).

[5] A. Zhao et al., Science 309, 1542 (2005).

[6] J. Park et al., Nature (London) 417, 722 (2002).

[7] W. Liang et al., Nature (London) 417, 725 (2002).

[8] L. H. Yu and D. Natelson, Nano Lett. 4, 79 (2004).

[9] L. H. Yu et al., Phys. Rev. Lett. 93, 266802 (2004).

[10] A. N. Pasupathy et al., Science 306, 86 (2004).

[11] V. Madhavan et al., Science 280, 567 (1998).

[12] H.C. Manoharan, C.P. Lutz, and D. M. Eigler, Nature (London) 403, 512 (2000).

[13] N. Knorr et al., Phys. Rev. Lett. 88, 096804 (2002).

[14] V. Madhavan et al., Phys. Rev. B 66, 212411 (2002).

[15] W. Chen, T. Jamneala, V. Madhavan, and M. F. Crommie, Phys. Rev. B 60, R8529 (1999).

[16] T. Jamneala, V. Madhavan, and M. F. Crommie, Phys. Rev. Lett. 87, 256804 (2001).

[17] T. Jamneala, V. Madhavan, W. Chen, and M. F. Crommie, Phys. Rev. B 61, 9990 (2000).

[18] J. Li, W.-D. Schneider, R. Berndt, and B. Delley, Phys. Rev. Lett. 80, 2893 (1998).

[19] M. A. Schneider, L. Vitali, N. Knorr, and K. Kern, Phys. Rev. B 65, 121406(R) (2002).

[20] P. Wahl et al., Phys. Rev. Lett. 93, 176603 (2004).

[21] K. Nagaoka, T. Jamneala, M. Grobis, and M. F. Crommie, Phys. Rev. Lett. 88, 077205 (2002).

[22] V. Madhavan et al., Phys. Rev. B 64, 165412 (2001).

[23] X. Lu and K. W. Hipps, J. Phys. Chem. B 101, 5391 (1997).

[24] M.-S. Liao and S. Scheiner, J. Chem. Phys. 114, 9780 (2001).

[25] The Fe atom has a significant $d$-orbital character near the Fermi level for $\mathrm{Fe}(\mathrm{II}) d^{6}$ system $[23,24]$. Our theoretical calculations, based on the stable configurations in Fig. 3, reveal that the highest occupied molecular orbital and the lowest unoccupied molecular orbital are located at $-1.26 \mathrm{eV}$ below the Fermi level and $1.01 \mathrm{eV}$ above the Fermi level, respectively.

[26] U. Fano, Phys. Rev. 124, 1866 (1961).

[27] O. Újsághy, J. Kroha, L. Szunyogh, and A. Zawadowski, Phys. Rev. Lett. 85, 2557 (2000).

[28] T. Kawasaka, H. Kasal, W. A. Dino, and A. Okiji, J. Appl. Phys. 86, 6970 (1999).

[29] A. Schiller and S. Hershfield, Phys. Rev. B 61, 9036 (2000).

[30] M. Plihal and J.W. Gadzuk, Phys. Rev. B 63, 085404 (2001).

[31] P. S. Cornaglia and C. A. Balseiro, Phys. Rev. B 67, 205420 (2003).

[32] J. Merino and O. Gunnarsson, Phys. Rev. B 69, 115404 (2004).

[33] J. P. Perdew et al., Phys. Rev. B 46, 6671 (1992).

[34] P. E. Blöchl, Phys. Rev. B 50, 17953 (1994).

[35] G. Kresse and D. Joubert, Phys. Rev. B 59, 1758 (1999).

[36] G. Kresse and J. Furthmüller, Phys. Rev. B 54, 11169 (1996).

[37] G. Kresse and J. Hafner, Phys. Rev. B 47, R558 (1993).

[38] B. Johnson, P. M. W. Gill, and J. A. Pople, J. Chem. Phys. 98, 5612 (1993).

[39] D. X. Shi et al., Phys. Rev. Lett. 96, 226101 (2006). 